\title{
Evaluation of controlled hydroxychloroquine releasing performance from calcium-alginate beads
}

\author{
Canan Armutcu (iD) Sena Piskin (iD \\ Hacettepe University, Department of Chemistry, Ankara, Turkey
}

\section{A BSTRACT}

\begin{abstract}
This study aimed to develop an effective controlled drug delivery system based on al1 ginate beads to treat autoimmune diseases such as Rheumatoid Arthritis (RA) and Systemic Lupus Erythematosus (SLE). The present study describes the drug delivery systems to control the practical uses of hydroxychloroquine (HCQ) by Ca-alginate beads. The characterization techniques were employed to evaluate the physicochemical properties as scanning electron microscopy (SEM), swelling test (S), hydrolytic degradation (weight loss, WL), and Fourier transform infrared-attenuated total reflection (FTIR-ATR). The release studies from alginate beads prepared in various drug doses were carried out in the aqueous solutions at different $\mathrm{pH}(5-8)$ and temperatures $\left(4-37^{\circ} \mathrm{C}\right)$. The approximately half-amount of HCQ in HCQ-AB3 was released in $12 \mathrm{~h}$, and about $84.38 \%$ was released within 8 days. Korsmeyer-Peppas was applied to model the HCQ release kinetic of alginate beads, which corresponded to the non-Fickian transport mechanism.
\end{abstract}

Keywords:

Calcium-alginate beads; hydroxychloroquine; drug delivery; RA; SLE

\author{
Article History: \\ Received: 2021/06/25 \\ Accepted: 2021/08/31 \\ Online: $2021 / 09 / 29$
}

Correspondence to: Canan Armutcu, Hacettepe University, Faculty of Science, Department of Chemistry, TR-06800 Ankara, Turkey

E-Mail: cananarmutcu@hacettepe.edu.tr

Phone: +90 (312) 2977963

Fax: $+90(312) 2992163$

\section{INTRODUCTION}

A ginate is a well-known and most favored biomaterial found in many applications such as the food sector, textile industry, waste removal, and pharmaceutical industries [1-4]. Alginate is a polysaccharide composed of D-mannuronic acid and $L$-guluronic acid monomers $[5,6]$. One of the essential properties of alginate is its ability to react with divalent cations and form insoluble crosslinked gels due to the carboxyl groups in the structure. Still, the standard method has been calcium ion gelation to obtain alginate beads $[7,8]$. Calcium alginate is the most commonly employed system for its easiness of gel formation. Once liquid alginate solutions are contacted with polycation $\left(\mathrm{Ca}^{2+}\right)$, they are immediately transformed into gel binding between guluronic acid blocks in alginate and $\mathrm{Ca}^{2+}$. Ca-Alginate beads are widely used in drug delivery due to their favorable properties such as non-toxic, water-soluble, film-forming, biocompatibility, ease of gelation, and biodegradable [9-11].

Controlled drug release is the technique that provides releasing of a drug at the therapeutic dose to the desired site in the body $[12,13]$. The importance of intelligent drug delivery systems (DDSs) in biomedical and pharmacological fields is growing daily. The main goal of DDSs is to provide drug release for long periods avoiding the side effects of the drugs, that increase the efficacy and safety of drug. The delivery system will also be biocompatible or biodegradable so that it is transformed into non-toxic parts that are eliminated harmlessly from the body $[14,15]$.

Chloroquine (CQ) and its derivate hydroxychloroquine (HCQ) are classified as anti-malarial agents [16]. Although, during the Second World War, the use of anti-malarial as prophylaxis by millions of soldiers, these drugs have been shown for the therapeutic effects in numerous other autoimmune diseases such as Rheumatoid Arthritis (RA) and Systemic Lupus Erythematosus (SLE) $[17,18]$. HCQ is widely used as it effectively controls dermatological complications in SLE, an autoimmune disease. However, it has been reported to effectively manage the symptoms of Sjøgren syndrome, a chronic autoimmune disease, and prevent thrombosis in phospholipid antibody (aPL) syndrome. It has been observed that HCQ also reduces the risks of atherosclerosis and cardiovascular disease in RA patients [19]. In RA and SLE, HCQ is preferred over CQ owing to the lower incidence of gastrointestinal adverse reactions and its safer dose-dependent toxicity profile [19-21]. HCQ has known controlling inflammatory processes and immunomodulatory effects. In addition, HCQ has a modulating effect on activated immune cells $[17,22]$. HCQ has also been widely used worldwide as a candida- 
te drug against SARS-CoV-2 infection to treat patients with COVID-19 due to its antiviral effects [23]. This has led to drug shortages and access problems for patients with SLE in many countries and rising anxiety, concerns in patients with SLE [24].

This study is focused on the treatment strategy to a controlled release of HCQ as a model therapeutics. If HCQ is taken in proper doses, it is a safe drug, although its safety margin is narrow, and a single higher dose might be fatal [17] Therefore, there is a need for randomized, controlled trials with this drug to prove its efficiency and safety for SLE and RA patients due to the potential for drug-induced toxicity.

This study aimed to develop a controlled drug release system for HCQ, selected as a therapeutic model. At the same time, alginate-based beads were chosen as a carrier system. First, alginate was crosslinked with the presence of calcium ions by a peristaltic pump at room temperature for $1 \mathrm{~h}$. Then, obtained alginate beads were characterized by scanning electron microscopy (SEM), swelling test (S), hydrolytic degradation (weight loss, WL) and Fourier transform infrared-attenuated total reflection (FTIR-ATR). The drug delivery experiments were studied to assure safety and appropriate dose use to include well-designed clinical trials in treating SLE and RA disease.

\section{MATERIAL AND METHOD}

\section{Materials}

Alginic acid sodium salt with medium viscosity (from brown algae) and hydroxychloroquine sulfate were purchased from Sigma-Aldrich (St. Louis, MO, USA). Calcium chloride dihydrate was obtained from Merck (Darmstadt, Germany). All other reagents were analytical grade and obtained from Merck.

\section{Preparation of HCQ loaded alginate beads}

Sodium alginate $(1 \%, \mathrm{w} / \mathrm{v})$ was dissolved in deionized water. The alginate solution was stirred to obtain a homogeneous solution without any bubbles at room temperature. Then, the homogeneous mixture was added dropwise into $\mathrm{CaCl}_{2}$ solution $(5 \%, \mathrm{w} / \mathrm{v})$ via peristaltic pump $(1 \mathrm{~mL} /$ min, flowrate). The obtained spherical alginate beads were kept in the crosslinking solution for $20 \mathrm{~min}$. After filtering the beads, they were transferred into pure ethanol for post-crosslinking/gelation purposes for $20 \mathrm{~min}$ and subsequently washed with deionized water. Finally, spherical alginate beads were placed on petri dishes and dried under two different conditions as room temperature and lyophilization. Drug-loaded alginate beads were prepared via the approach mentioned above with HCQ in feeding solution in different amounts of 0.05, 0.1, and 0.2 g. According to the three different drug content, the alginate beads were labeled as HCQ-AB1, HCQ-AB2 and HCQ-AB3, respectively.

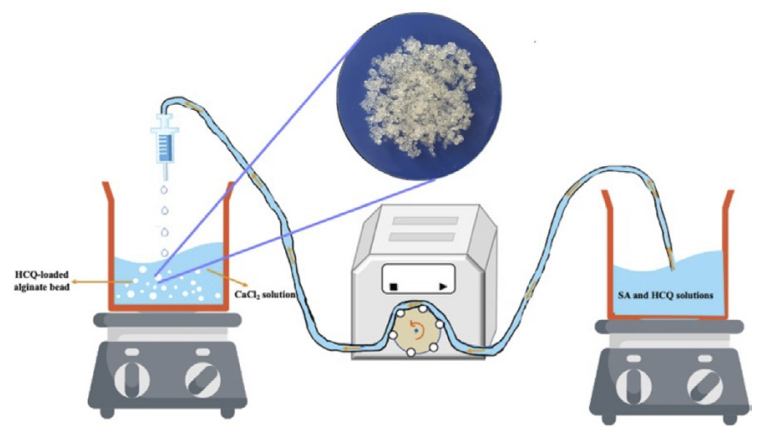

Figure 1. Schematic representation for the preparation of HCQ loaded alginate beads.

\section{Characterization of Alginate Beads}

The prepared alginate beads were characterized by the following techniques. The equilibrium swelling degrees of both freeze-dried and air-dried alginate beads were determined by immersing the dried beads $(30 \mathrm{mg}$ ) into PBS ( $\mathrm{pH} 7.4,10 \mathrm{~mL}$ ). The swollen beads were removed from the buffer solution, quickly wiped out with a filter paper, and reweighed $\left(\mathrm{W}_{\mathrm{s}}\right)$ at defined time intervals. The beads weights (dried and wet) were recorded to calculate the percentage swelling rate of beads by the below equation:

$$
\mathrm{S} \%=\left[\mathrm{W}_{\mathrm{s}}-\mathrm{W}_{0}\right] \times 100
$$

Herein, $W_{0}$ and $W_{\mathrm{s}}$ designated the weights (g) of beads before and after swelling, respectively.

The hydrolytic degradation behavior was also characterized by investigating the weight loss of freeze-dried alginate beads. The initially beads were immersed in PBS $(\mathrm{pH}$ $7.4,10 \mathrm{~mL}$ ) and incubated by using a temperature-controlled shaking-bath at $37^{\circ} \mathrm{C}$ at $100 \mathrm{rpm}$ for 8 days. The alginate beads were removed from the PBS and weighted at different intervals. After alginate beads were dried, the hydrolytic degradation behavior was examined. The hydrolytic degradation rate of the developed alginate beads was determined as the percentage of weight loss (WL) by the following equation:

$$
\mathrm{WL} \%=\left[\left(\mathrm{W}_{0}-\mathrm{W}_{\mathrm{t}}\right) / \mathrm{W}_{0}\right] \times 100
$$

where $\mathrm{W}_{0}$ is the weight of the initial dry sample and $\mathrm{W}_{\mathrm{t}}$ is the weight of the dry sample for a given time $t$.

To assess the morphology of dried beads, SEM images were obtained with a GAIA3 (Tescan, Czech Republic) utilized from Hacettepe University, Advanced Technologies 
Application and Research Center (HUNITEK, Ankara, Turkey). The dried beads were coated with gold and scanned by SEM with different magnifications at a high vacuum. In addition, FTIR-ATR was conducted to characterize the functional groups of alginate beads before and after the degradation process. The FTIR-ATR (Perkin Elmer, Spectrum $\mathrm{Two}^{\mathrm{TM}}$ ) spectrum was recorded over the wavenumber range of $4000-400 \mathrm{~cm}^{-1}$.

\section{HCQ encapsulation efficiency}

HCQ encapsulation efficiency was investigated by dividing the trapped HCQ amount in the alginate beads by the total amount of HCQ in the alginate solution. The residual HCQ in the $\mathrm{CaCl}_{2}$ gelling and washing solutions was collected and measured by UV/Vis spectrophotometer at $342 \mathrm{~nm}$. HCQ encapsulation efficiency of alginate beads was calculated using the following equation:

$$
\text { Encapsulation Efficiency }(\mathrm{EE})=\frac{\text { total HCQ }- \text { residual HCQ }}{\text { total HCQ }}
$$

\section{In vitro $H C Q$ release studies}

The drug-loaded alginate beads with a different weight (20-50 mg) were placed into a glass bottle containing 4 $\mathrm{mL}$ buffer solution in the $\mathrm{pH}$ range of 5.0-8.0 and at different temperatures $\left(4-37^{\circ} \mathrm{C}\right)$. The drug release studies were performed in an incubator shaker at $100 \mathrm{rpm}$ (JSSB30T, JSR, Gongju, Korea). At the defined time intervals, $0.4 \mathrm{~mL}$ of the buffer solution was taken out and replaced with fresh buffer solution. The released HCQ from the alginate beads was determined at $342 \mathrm{~nm}$ using a UV/Vis spectrophotometer (Shimadzu, UV-1280, Tokyo, Japan). For statistical purposes, all the drug release procedures were performed in three replicates to calculate the standard deviation.

\section{RESULTS AND DISCUSSION}

\section{Characterization studies}

—igure 2 shows the swelling ratios of both the free- ze-dried and air-dried HCQ-loaded alginate beads in buffer solution at $\mathrm{pH}$ 7.4. As shown in the figure, the freeze-dried alginate beads performed higher swelling degrees than the air-dried alginate beads in the equilibrium. The freeze-dried alginate beads showed a moderate swelling degree of $3088 \%$ at 6 days, while air-dried alginate beads reached $1142 \%$. These results clearly showed that freeze-dried samples have a higher porosity structure than air-dried alginate beads. In other words, the air-dried alginate beads had a more compact and dense structure than freeze-dried ones. This may be due to the high speed of solvent removal at a high vacuum in the

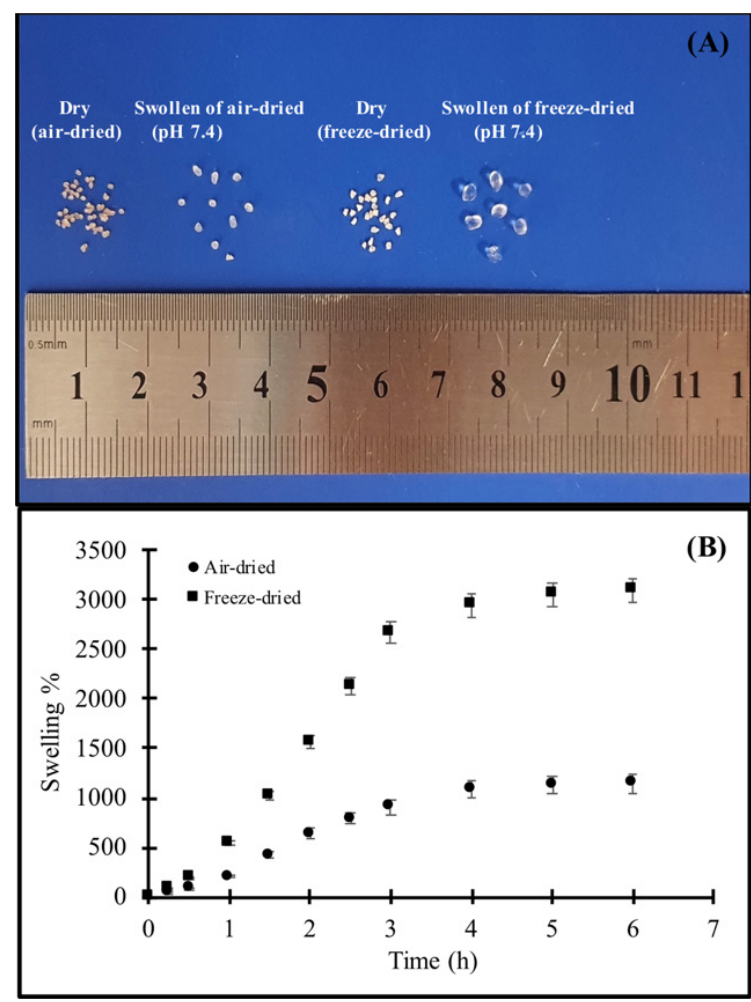

Figure 2. Optical images of the prepared dried HCQ-loaded alginate beads (air-dried and freeze-dried) and swollen sizes of air-dried and freeze-dried HCQ-loaded alginate beads in $\mathrm{pH} 7.4$ (A) and swelling degrees of both the freeze-dried and air-dried HCQ-loaded alginate beads in $\mathrm{pH}$ 7.4 at $37^{\circ} \mathrm{C}(\mathrm{B})$.

freeze-drying method. In the air-drying method, solvent removal occurs slowly, leading to a less porous structure of the alginate beads [25].

To measure the hydrolytic degradation behavior of the freeze-dried HCQ-loaded alginate beads, they were placed in PBS (pH 7.4) at $37^{\circ} \mathrm{C}$ for 8 days (Figure 3). The weight loss of alginate beads gradually increased concerning the time as $27.6 \%$ after 8 days at $\mathrm{pH} 7.4$ because of the hydrolytic degradation. Thus, it was shown that the freeze-dried HCQloaded alginate beads were exhibited a long-term degradation behavior with good stability that makes the prepared alginate beads a good alternative for DDSs.

SEM was used to characterize the morphologies of air and freeze-dried HCQ-loaded alginate beads. The drying method had a significant effect on the beads' regularity and surface morphology. As shown in Figure 4, the air-dried beads (4A) appear more compact, dense, and smoother than freeze-dried beads (4B). Freeze-dried beads had relatively larger sizes and highly porous structures in comparison with air-dried beads due to the lyophilization procedure. The enclosed water in the beads was quickly removed without major deteriorate of the pores during lyophilization. The figure shows that there were relatively rough surfaces and many cavities in the freeze-dried beads integral regions 


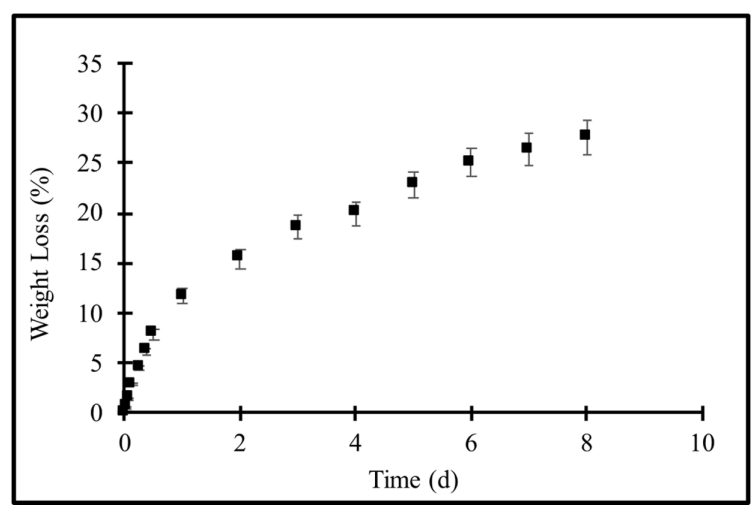

Figure 3. Hydrolytic degradation of HCQ-loaded alginate beads in $\mathrm{pH}$ 7.4 at $37^{\circ} \mathrm{C}$.

(4D). The porous structure of the freeze-dried beads is suitable for drug delivery due to provide easy penetration of buffer solution into the structure $[25,26]$.

The morphological changes of degraded alginate beads after incubated for 8 days in $\mathrm{pH} 7.4$ at $37^{\circ} \mathrm{C}$ were also investigated by SEM. There was not any agglomeration in the structure of alginate beads before incubation in $\mathrm{pH} 7.4$ (Figure 5B). The changes of alginate beads structure were observed as soon as incubation was started with the $\mathrm{pH} 7.4$. The morphological changes were observed at alginate beads after $6 \mathrm{~h}$ (Figure 5C), $24 \mathrm{~h}$ (D) and, 8 days (E, F). As the incubation continued, the rate of degradation was increased, and more conglutination, shrinkages, and the mass loss of the structure of alginate beads were seen (Figure 5E-F). The weight loss of HCQ loaded alginate beads reached 27.6\% at 8 days. The SEM images of degraded alginate beads after incubation were evidence of the weight loss results of alginate beads.

Functional groups of $\mathrm{HCQ}$, alginate beads, and HCQ loaded alginate beads were determined using FTIR-ATR spectrometer (Figure 6). The main characteristics bands for $\mathrm{HCQ}$ (Figure 6A), -OH stretching peaks were observed at $3200 \mathrm{~cm}^{-1}$ and $\mathrm{NH}$ deformations bands at approximately $3300 \mathrm{~cm}^{-1}$. Moreover, the aromatic $\mathrm{C}=\mathrm{C}$ stretching bands at 1450 and $1610 \mathrm{~cm}^{-1}$ and $\mathrm{C}-\mathrm{N}$ bands at around $1100 \mathrm{~cm}^{-1}$ occurred in the spectrum. Similarly, the $\mathrm{C}-\mathrm{Cl}$ stretching peak is located between 550 and $850 \mathrm{~cm}^{-1}$. The characteristic peaks of HCQ disappear in the HCQ-loaded beads because of the overlap of bands in this region comprising the deformation of $-\mathrm{OH},-\mathrm{NH}$, and $-\mathrm{CH}$. Moreover, there is a decrease in the intensity of $1100 \mathrm{~cm}^{-1}$ and $1450 \mathrm{~cm}^{-1}$ because of the strong bond stretches of the alginate chain covering the HCQ drug [27]. On the other hand, the spectrum of HCQ loaded alginate beads almost matched up with the spectrum of alginate beads. Only one common peak was detected in HCQ and HCQ-loaded beads different from alginate beads at $1280 \mathrm{~cm}^{-1}$, which was exhibited the presence of the $\mathrm{C}-\mathrm{O}$ stretch. In addition, when the FTIR-ATR spectra of alginate beads were investigated before and after the degradation process (6h, 24h, and 8 days), characteristic peaks intensity was decreased around 1600, 1418, and 1030 $\mathrm{cm}^{-1}$, indicating the asymmetric stretching vibration and symmetric stretching vibration of the $-\mathrm{COO}-$ group and, $\mathrm{C}-\mathrm{O}-\mathrm{C}$ stretching vibrations, respectively (corresponding figures were also given in Supplementary File, Figure SI-1).

\section{Encapsulation of Hydroxychloroquine by alginate beads}

Encapsulation efficiencies of HCQ loaded alginate beads were calculated by considering the crosslinking and washing processes. The encapsulation efficiencies of HCQ-AB1, HCQ-AB2, and HCQ-AB3 were calculated as $43.98 \%, 55.77 \%, 81.22 \%$, respectively. In addition, it was observed an increase in EE with increasing the concentration of HCQ in the alginate beads. In the light of these results, HCQ-AB3 labeled beads was used in all release studies.

\section{HCQ release studies}

For the optimization studies, the effects of medium $\mathrm{pH}$, drug content, beads amount, and temperature were investigated on the alginate beads. The effect of medium $\mathrm{pH}$ on HCQ delivery was performed in the appropriate ranges of $\mathrm{pH}$ 5.0-8.0, as shown in Figure 7. The release profiles of HCQ from alginate beads exhibited a biphasic delivery system. It was observed to release HCQ at different rates, initial burst release step within 12 hours followed by a constant rate (ideally) and sustained release. As observed in Figure 7., the medium pH significantly affected the HCQ release properties. As seen from Figure $7 \mathrm{~A}$, the amount of $\mathrm{HCQ}$ released at $\mathrm{pH} 5.0$ was relatively low and only about $9.5 \%$, while this value was $24.5 \%$ for $\mathrm{pH} 7.4$ within $60 \mathrm{~min}$. The low drug release ratio is due to the shrinkage and less swelling of the alginate beads in acidic buffer [1]. HCQ release amounts were $14.48 \%$, $17.89 \%, 23.89 \%, 42.36 \%$, and $30.92 \%$ in $12 \mathrm{~h}$ at $\mathrm{pH}$ values of 5.0, 6.0, 7.0, 7.4 and 8.0, respectively. After the immediate releasing phase, the cumulative release was $84.38 \%$ at $\mathrm{pH} 7.4$ after $192 \mathrm{~h}$.

The cumulative release of HCQ from alginate beads at different amounts of HCQ loadings is seen in Figure 8. It was seen that the cumulative release of HCQ was increased from 38.76 to $84.38 \%$ with the increasing amount of HCQ from 50 to $200 \mathrm{mg}$. Moreover, the release profile of drugs from HCQ-AB3 realized fast and higher release rates about 2.5 -fold than HCQ-AB1 within $12 \mathrm{~h}$. The higher drug amount in the alginate beads resulted in the faster diffusion rate of HCQ from the alginate beads, which cause a higher release rate of $\mathrm{HCQ}$, especially in the early stage of the release 


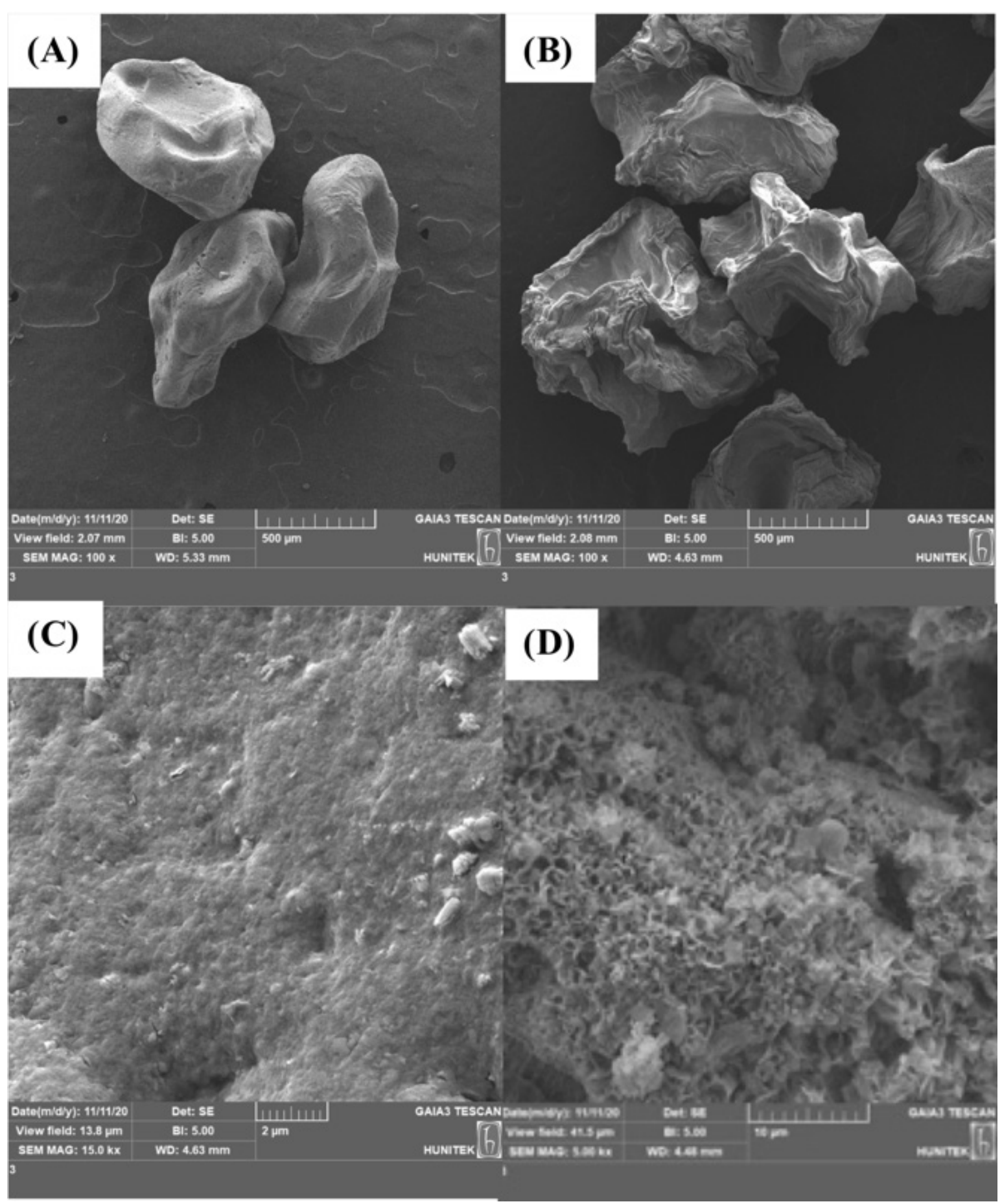

Figure 4. SEM images of air-dried (A, C) and freeze-dried HCQ-loaded alginate beads (B, D).

process [28]. Another reason for the increase in the amount of released drugs is that the drug delivery systems are swelling controlled. Therefore, when the alginate begins to absorb water, it causes a more significant amount of drug diffuses in the mass of the swelling bath [29].

The effect of temperature on the HCQ release profile was carried out at 4,25 , and $37^{\circ} \mathrm{C}$, and HCQ release data was presented in Figure 9. The HCQ release was $84.38 \%$ af- ter 8 days at $37^{\circ} \mathrm{C}$, whereas the value was less than $32.52 \%$ at $4^{\circ} \mathrm{C}$ with the same treatment. The increasing temperature promotes the penetration of buffer solution into the beads, which enhances swelling of alginate sites in beads and causes the squeezing out of the drug [30].

The effect of HCQ-AB3 bead amounts on the cumulative release was determined by using various bead amounts (20-50 mg beads). As seen from Figure 10, the maximum 


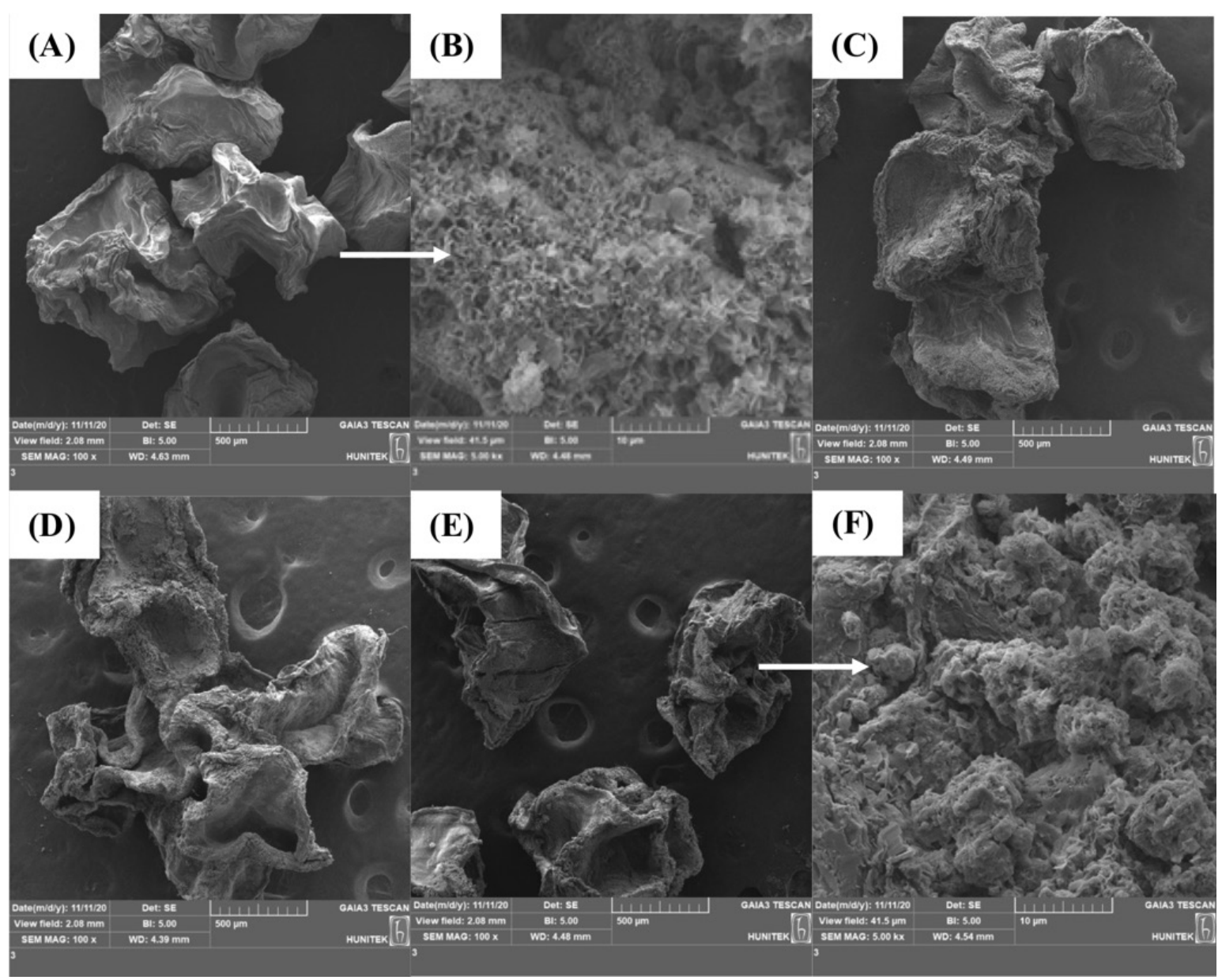

Figure 5. SEM images of freeze-dried HCQ loaded alginate beads before and after incubation at $37^{\circ} \mathrm{C}$ in $\mathrm{pH}$ 7.4. Freeze-dried HCQ loaded alginate beads (A, B), after 6 hours (C), after 24 hours (D), after 8 days (E, F).

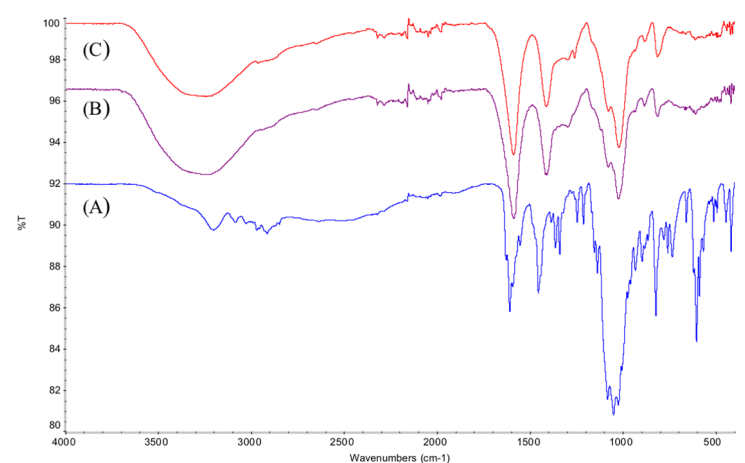

Figure 6. FTIR-ATR spectra of HCQ (A), alginate beads (B), and HCQ loaded alginate beads $(\mathrm{C})$

cumulative release was realized with $30 \mathrm{mg}$ of HCQ-AB3 alginate beads both the first $12 \mathrm{~h}$ and 8 days. The lower cumulative releases of HCQ were obtained at high bead amounts. The amount of the beads and the dose of the drug in the beads are significant to show the effect of the released active substance. Undesirable effects may occur when the dose rate falls below the sufficient amount or rises above the toxic level. The result figured out that the amount of beads to be used is significant for controlling the release performances.

\section{Release kinetic studies of HCQ}

To understand the release profile of HCQ from HCQAlginate beads, the release data were analyzed using a classic empirical equation:

$$
\frac{\mathrm{M}_{\mathrm{t}}}{\mathrm{M}_{\circ}}=\mathrm{kt}^{\mathrm{n}}
$$

where $M_{t}$ means the amount of drug release at time $t$, $M_{\infty}$ describes the amount of drug release at an infinite time, $\mathrm{k}$ is the release kinetic constant at the Korsmeyer-Peppas model. The release exponent is $n$ which is related to the geometry of the release system and indicates the release mechanism. The Korsmeyer-Peppas equation can adequately describe the release of drugs from spheres [31,32]. When the value of 0.43 or less than 0.43 , the release mechanism is based on diffusion (Fickian diffusion or quasi-Fickian diffusion). If the value of between 0.43 and 0.85 , the release mechanism is based on an anomalous diffusion or non-Fickian diffusion (i.e., a mixed diffusion, polymer relaxation). Moreover, the value above 0.85 , the release mechanism is based on a Case- 

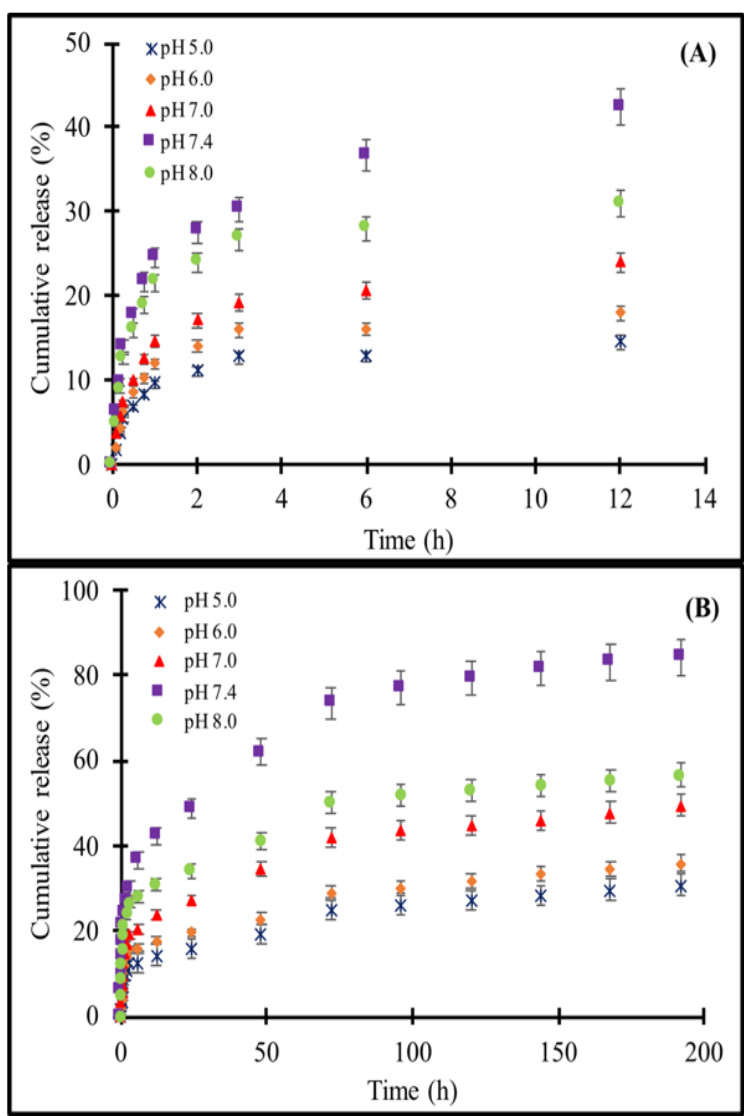

Figure 7. SEM images of freeze-dried HCQ loaded alginate beads before and after incubation at $37^{\circ} \mathrm{C}$ in $\mathrm{pH}$ 7.4. Freeze-dried HCQ loaded alginate beads $(A, B)$, after 6 hours $(C)$, after 24 hours (D), after 8 days (E, F).

II transport related to polymer relaxation on the drug molecules movement during gel swelling [33,34]. The correlation coefficient $\left(R^{2}\right)$ was used to evaluate the fitness of the model.

Table 1 presented the release exponent (n) values and correlation coefficient $\left(\mathrm{R}^{2}\right)$ for HCQ-alginate beads. The exponent values $(0.43<\mathrm{n}<0.85)$ demonstrated that the drug release mechanism was anomalous diffusion for each bead. According to the Korsmeyer-Peppas model, the drug release mechanism of HCQ-Alginate beads observed both diffusion and swelling controlled drug release (anomalous diffusion) $[33,35]$. In general, the release kinetics of alginate beads fitted well with the Korsmeyer-Peppas model $\left(R^{2}>0.97\right)$.

\section{CONCLUSIONS}

In this study, HCQ was encapsulated in alginate beads to use a controlled drug release system. The obtained data demonstrated a significant effect of the drying method on the characteristics of the prepared alginate beads. The highest HCQ encapsulation efficiency (84.38\%) was obtained with the HCQ-AB3 formulation, which exhibited biphasic delivery systems at different rates to assure appropriate dose use and safety. Alginate beads loaded with HCQ showed drug release for 8 days with a moderate
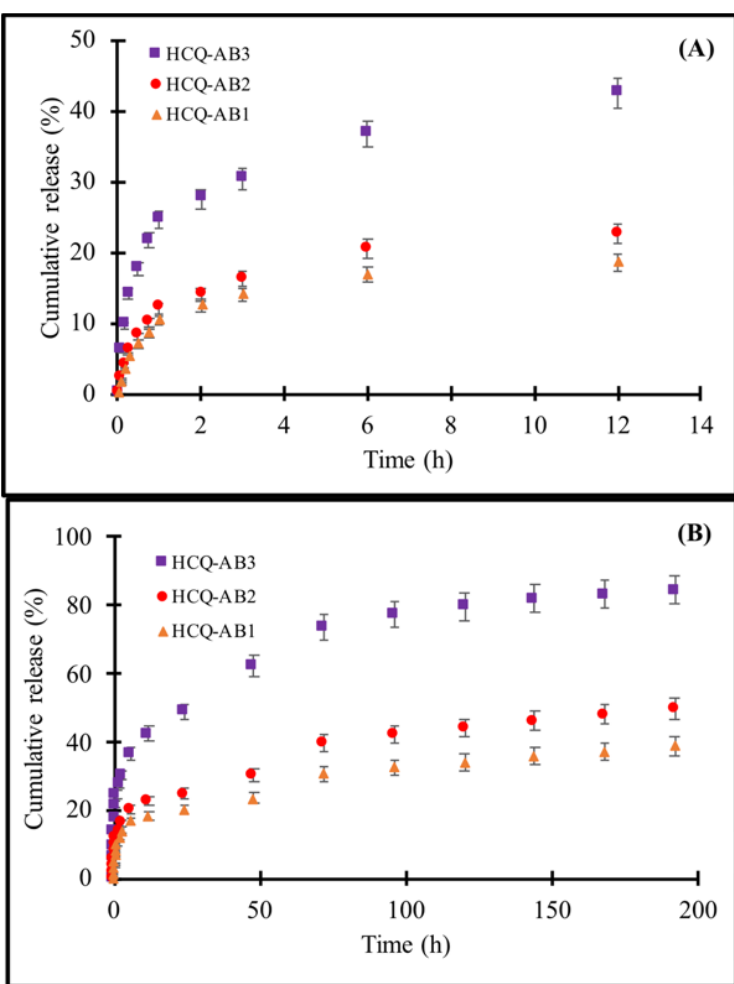

Figure 8. Cumulative release of $\mathrm{HCQ}$ from alginate beads containing different drug amounts at $\mathrm{pH} 7.4$ at $37^{\circ} \mathrm{C}$. HCQ release (A) for $12 \mathrm{~h}$ and (B) for 8 days
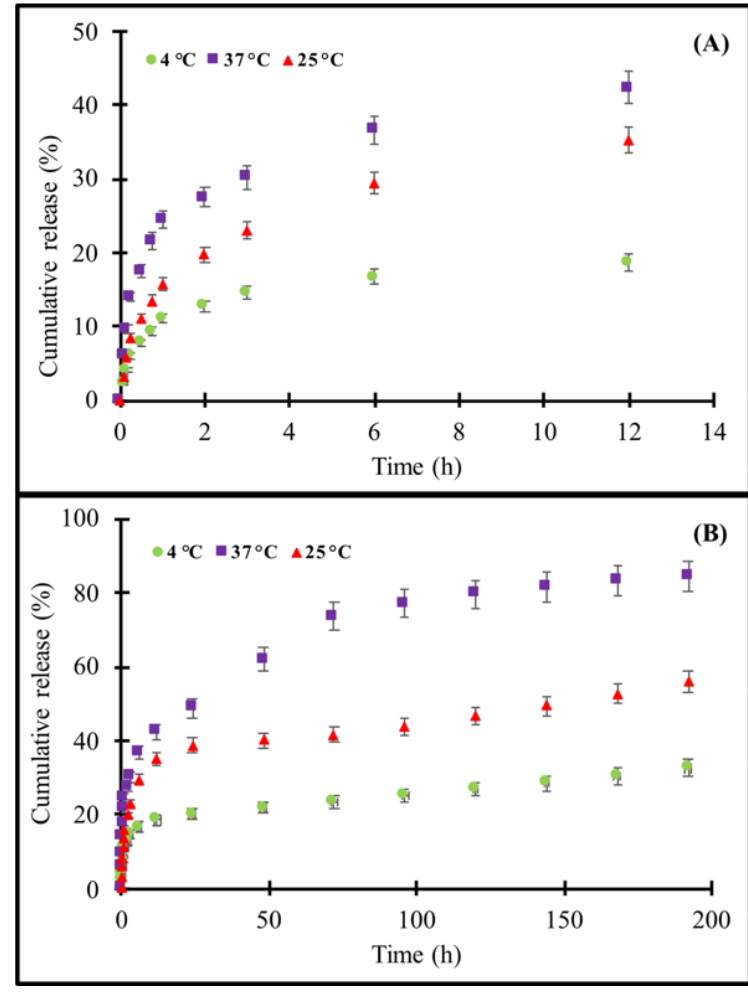

Figure 9. Cumulative release of HCQ from HCQ-AB3 at a different temperature at $\mathrm{pH}$ 7.4. HCQ release (A) for $12 \mathrm{~h}$ and (B) for 8 days.

burst release of $42.36 \%$ within 12 h. Korsmeyer-Peppas model showed that the drug release mechanism was ano- 


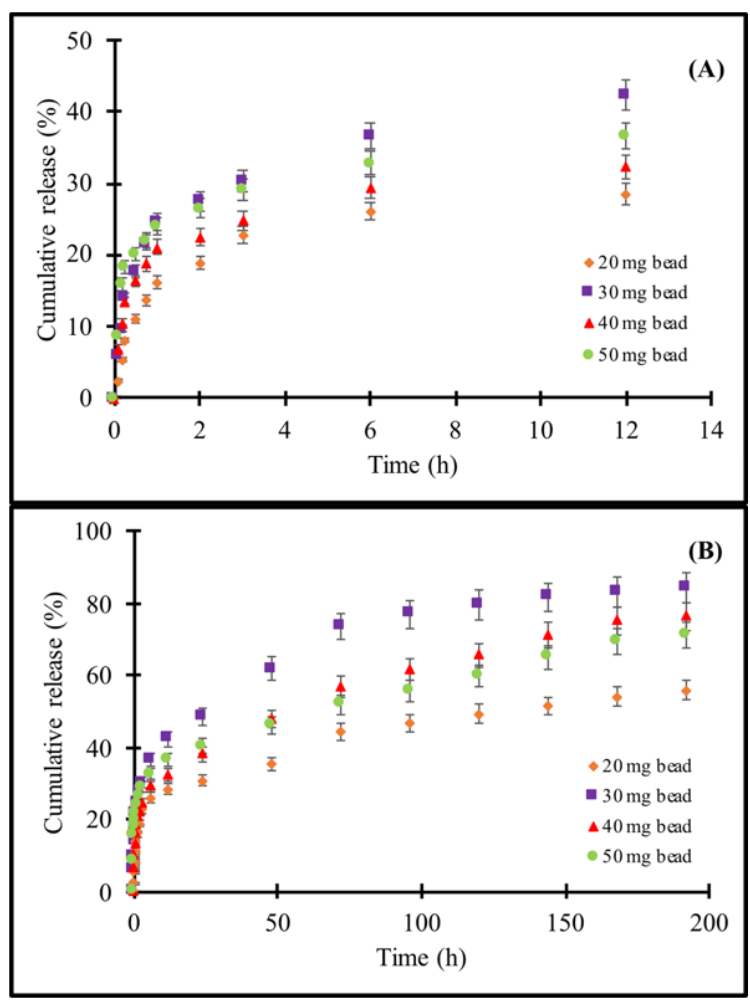

Figure 10. Cumulative release of HCQ from HCQ-AB3 at various bead amounts at $\mathrm{pH} 7.4$ at $37^{\circ} \mathrm{C}$. HCQ release (A) for $12 \mathrm{~h}$ and (B) for 8 days.

Table 1. Values of the release exponent (n) and correlation coefficients $\left(\mathrm{R}^{2}\right)$ for HCQ-Alginate beads at $\mathrm{pH} 7.4$ at $37^{\circ} \mathrm{C}$.

\begin{tabular}{lcc}
\hline Polymer code & $\mathbf{n}$ & $\mathbf{R}^{2}$ \\
\hline HCQ-AB1 & 0.7332 & 0.9774 \\
HCQ-AB2 & 0.6936 & 0.9897 \\
HCQ-AB3 & 0.5587 & 0.9969 \\
\hline
\end{tabular}

malous diffusion, i.e., $\mathrm{HCQ}$ could release at $\mathrm{pH} 7.4$ in a controlled manner.

\section{CONFLICT OF INTEREST}

Authors approve that to the best of their knowledge, there is not any conflict of interest or common interest with an institution/organization or a person that may affect the review process of the paper.

\section{AUTHOR CONTRIBUTION}

Sena Piskin: Writing - original draft, Visualization, Investigation. Canan Armutcu: Supervision, Conceptualization, Writing - review \& editing.

\section{References}

1. Kaygusuz H, Erim F. Alginate/BSA/montmorillonite composites with enhanced protein entrapment and controlled release efficiency. Reactive \& Functional Polymers 73 (2013) 1420-1425.
2. Kaygusuz H, Torlak E, Akın-Evingür G, Özen İ, Von Klitzing R, Erim FB. Antimicrobial cerium ion-chitosan crosslinked alginate biopolymer films: A novel and potential wound dressing. International Journal of Biological Macromolecules 105 (2017) 1161-1165.

3. Tamahkar E, Özkahraman B, Özbaş Z, Izbudak B, Yarimcan F, Boran F, Öztürk AB. Aloe vera-based antibacterial porous sponges for wound dressing applications. Journal of Porous Materials 28 (2021) 741-750.

4. Hussain S, Abid MA, Munawar KS, Saddiqa A, Iqbal M, Suleman M, Hussain M, Riaz M, Ahmad T, Abbas A, Rehman M, Amjad M. Choice of Suitable Economic Adsorbents for the Reduction of Heavy Metal Pollution Load. Polish Journal of Environmental Studies 30 (2021) 1-11.

5. Jayakumar R, Rajkumar M, Freitas H, Selvamurugan N, Nair SV, Furuike T, Tamura H. Preparation, characterization, bioactive and metal uptake studies of alginate/phosphorylated chitin blend films. International Journal of Biological Macromolecules 44 (2009) 107111.

6. Liu C-m, He X-h, Liang R-h, Liu W, Guo WL, Chen J. Relating physicochemical properties of alginate-HMP complexes to their performance as drug delivery systems. Journal of Biomaterials Science, Polymer Edition 28 (2017) 2242-2254.

7. Alexander BR, Murphy KE, Gallagher J, Farrell GF, Taggart G. Gelation time, homogeneity, and rupture testing of alginatecalcium carbonate-hydrogen peroxide gels for use as wound dressings. Journal of Biomedical Materials Research Part B: Applied Biomaterials 100 (2012) 425-431.

8. Liparoti S, Speranza V, Marra F. Alginate hydrogel: The influence of the hardening on the rheological behaviour. Journal of the Mechanical Behavior of Biomedical Materials 116 (2021) 104341.

9. Gowri M, Latha N, Suganya K, Murugan M, Rajan M. Calcium alginate nanoparticle crosslinked phosphorylated polyallylamine to the controlled release of clindamycin for osteomyelitis treatment. Drug Development and Industrial Pharmacy 47 (2021) 280-291.

10. Lin N, Gèze A, Wouessidjewe D, Huang J, Dufresne A. Biocompatible double-membrane hydrogels from cationic cellulose nanocrystals and anionic alginate as complexing drugs codelivery. ACS Applied Materials \& Interfaces 8 (2016) 6880-6889.

11. Pongjanyakul T, Rongthong T. Enhanced entrapment efficiency and modulated drug release of alginate beads loaded with drugclay intercalated complexes as microreservoirs. Carbohydrate Polymers 81 (2010) 409-419.

12. Alvarez-Lorenzo C, Concheiro A. 15 Review of Smart Materials for Controlled Drug Release. Fundamentals of Smart Materials (2020) 170.

13. Ravi Kumar MN, Kumar $₫ N$. Polymeric controlled drug-delivery systems: perspective issues and opportunities. Drug Development and Industrial Pharmacy 27 (2001) 1-30.

14. Cunliffe D, Kirby A, Alexander C. Molecularly imprinted drug delivery systems. Advanced Drug Delivery Reviews 57 (2005) 18361853.

15. Lee E, Kim S, Seong K, Park H, Seo H, Khang G, Lee D. A biodegradable and biocompatible drug-delivery system based on polyoxalate microparticles. Journal of Biomaterials Science, Polymer Edition 22 (2011) 1683-1694.

16. da Silva AEA, de Abreu PMB, Geraldes DC, de Oliveira Nascimento L. Hydroxychloroquine: Pharmacological, physicochemical aspects and activity enhancement through experimental formulations. Journal of Drug Delivery Science and Technology 63 (2021) 102512.

17. Ben-Zvi I, Kivity S, Langevitz P, Shoenfeld, Y. Hydroxychloroquine: from malaria to autoimmunity. Clinical Reviews in Allergy \& 
Immunology 42 (2012) 145-153.

18. Walls AC, Park Y-J, Tortorici MA, Wall A, McGuire AT, Veesler D. Structure, function, and antigenicity of the SARS-CoV-2 spike glycoprotein. Cell 181 (2020) 281-292.

19. Rainsford K, Parke AL, Clifford-Rashotte M, Kean WF. Therapy and pharmacological properties of hydroxychloroquine and chloroquine in treatment of systemic lupus erythematosus, rheumatoid arthritis and related diseases. Inflammopharmacology 23 (2015) 231-269.

20. Jamalipour Soufi G, Iravani S. Potential inhibitors of SARS-CoV-2: recent advances. Journal of Drug Targeting 29 (2021) 349-364.

21. Tan YW, Yam WK, Sun J, Chu JJH. An evaluation of chloroquine as a broad-acting antiviral against hand, foot and mouth disease. Antiviral Research 149 (2018) 143-149.

22. Jorge AM, Melles RB, Zhang $\mathrm{Y}$, Lu N, Rai SK, Young LH, Costenbader KH, Ramsey-Goldman R, Lim SS, Esdaile JM, Clarke AE, Urowitz MB, Askanse A, Aranow C, Petri M, Choi H. Hydroxychloroquine prescription trends and predictors for excess dosing per recent ophthalmology guidelines. Arthritis Research \& Therapy 20 (2018) 1-8.

23. McKee DL, Sternberg A, Stange U, Laufer S, Naujokat C. Candidate drugs against SARS-CoV-2 and COVID-19. Pharmacological Research 157 (2020) 104859.

24. Cornet A, Andersen J, Tani C, Mosca M. Hydroxychloroquine availability during COVID-19 crisis and its effect on patient anxiety. Lupus Science \& Medicine 8 (2021) e000496.

25. El-Sherbiny IM, Abdel-Mogib M, Dawidar A-AM, Elsayed A, Smyth HD. Biodegradable pH-responsive alginate-poly (lacticco-glycolic acid) nano/micro hydrogel matrices for oral delivery of silymarin. Carbohydrate Polymers 83 (2011) 1345-1354.

26. George M, Abraham T. pH sensitive alginate-guar gum hydrogel for the controlled delivery of protein drugs. International journal of pharmaceutics 335 (2007) 123-129.
27. Moraes ANF, Silva LAD, de Oliveira MA, de Oliveira EM, Nascimento TL, Lima E M, Torres LMS, Diniz DGA. Compatibility study of hydroxychloroquine sulfate with pharmaceutical excipients using thermal and nonthermal techniques for the development of hard capsules. Journal of Thermal Analysis and Calorimetry 140 (2020) 2283-2292.

28. Çetin K, Alkan H, Bereli N, Denizli A. Molecularly imprinted cryogel as a $\mathrm{pH}$-responsive delivery system for doxorubicin. Journal of Macromolecular Science, Part A 54 (2017) 502-508.

29. Prabhakar S, Bajpai J, Bajpai AK, Tiwari A. Cumulative release of cefotaxim from interpenetrating networks of poly (vinyl alcoholg-acrylamide) and chitosan-g-polyacrylamide chains. Polymer Bulletin 71 (2014) 977-988.

30. Shi J, Zhang Z, Li G, Cao S. Biomimetic fabrication of alginate/ CaCO 3 hybrid beads for dual-responsive drug delivery under compressed CO 2. Journal of Materials Chemistry 21 (2011) 1602816034.

31. Ritger PL, Peppas NA. A simple equation for description of solute release I. Fickian and non-fickian release from non-swellable devices in the form of slabs, spheres, cylinders or discs. Journal of Controlled Release 5 (1987) 23-36.

32. Sanson C, Schatz C, Le Meins J-F, Soum A, Thévenot J, Garanger E, Lecommandoux S. A simple method to achieve high doxorubicin loading in biodegradable polymersomes. Journal of Controlled Release 147 (2010) 428-435.

33. Pasparakis G, Bouropoulos N. Swelling studies and in vitro release of verapamil from calcium alginate and calcium alginate-chitosan beads. International Journal of Pharmaceutics 323 (2006) 34-42.

34. Ritger PL, Peppas NA. A simple equation for description of solute release II. Fickian and anomalous release from swellable devices. Journal of Controlled Release 5 (1987) 37-42.

35. Peppas N. Analysis of Fickian and non-Fickian drug release from polymers. Pharmaceutica Acta Helvetiae 60 (1985) 110-111. 